JIOM Nepal, Volume 41, Number 2, August 2019, page 17-20

\title{
Mortality in Emergency Services in a University Teaching Hospital: A Retrospective Study
}

\author{
${ }^{1}$ Tirtha M Shrestha, 'Ramesh P Aacharya, 'Ram P Neupane, ²Bigyan Prajapati \\ 1Department of General Practice and Emergency Medicine, Maharajgunj Medical Campus, Institute of Medicine, \\ Tribhuvan University Teaching Hospital, Kathmandu, Nepal ${ }^{2}$ Kalikot District Hospital, Kalikot
}

\section{Corresponding author:}

Tirtha M Shrestha, MBBS, MD

Department of General Practice and Emergency Medicine, Maharajgunj Medical Campus, Institute of Medicine, Tribhuvan University Teaching Hospital, Kathmandu, Nepal

Email: tirtha_48@yahoo.com.au

Submitted: Jun 7, 2019

Accepted : Jul 14, 2019

\begin{abstract}
Introduction

Emergency services are the gateway between the community and hospital that provides 24-hour access for most needy patients in critical and emergency conditions. Mortality rate varies in emergency department across the world and even in different emergency units of the same hospital. This retrospective study was done in adult emergency services of a tertiary hospital to determine mortality rate and analyze causes of death.

\section{Methods}

A retrospective observational study of mortality cases to analyze mortality rate and causes of death of patients for a period of 6 months between October 2017 to March 2018 was carried out in the adult emergency services of Tribhuvan University Teaching Hospital, Kathmandu. Data required were collected from copies of death certificates.

\section{Results}

During the study period, a total of 128 patients died in emergency, accounting $0.5 \%$ of total patient. Male deaths (52.3\%) were slightly higher compared to female deaths (47.7\%). Age group 66-75 years had the highest (24.2\%) of total mortalities in the emergency. The most common immediate cause of death was sepsis/septic shock (21.9\%) followed by cardiopulmonary arrest, aspiration, respiratory failure, other causes of shock and poisoning. The commonest antecedent cause of death was attributed to respiratory causes. Similarly, the most common contributory cause of death was chronic obstructive pulmonary disease.

\section{Conclusion}

Older age group are prone to the mortality risk. Sepsis/septic shock was the most common immediate cause of death. Pneumonia was the most common antecedent causes of death. Chronic obstructive pulmonary disease was the commonest contributory cause.
\end{abstract}

Keywords: Emergency Department, mortality, Nepal, sepsis

\section{INTRODUCTION}

ए il mergency Department (ED) remains the gateway between community and hospital for critically ill patients and other emergency patients. Therefore, it represents the first place where many major diseases are presented. The ED undoubtedly plays an important role in the Nepalese health care system, dealing with minor injuries, trauma and acute medical/surgical and gynecological diseases. The management of patients in the emergency department is not uniform worldwide and depends upon the type of hospital setting, and the available human and physical resources. Due to high patient flow, limited manpower and infrastructure, poor financial status and the inability to obtain specialist care in time, a large number of patients with serious illnesses die in ED.

According to a United State's study, from 1998 to 2000 , the death rate in the emergency department increased by $26 \%,{ }^{1}$ and another study in Iran reported that the hospital mortality rate in the emergency department was as high as $60 \% .^{2}$ Determining the cause of ED death will help to plan to stop such events in the ED in the future. If the main cause of 


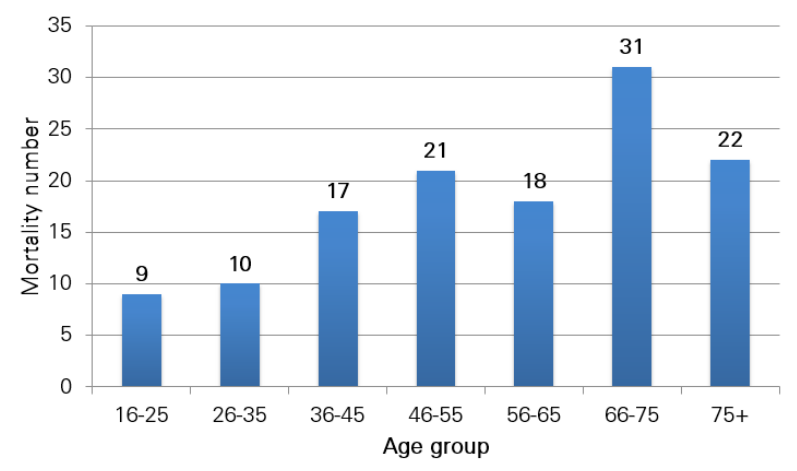

Figure 1. Age wise distribution of mortality

death is known, we can prioritize such cases from the next time. The $10^{\text {th }}$ revision of the International Classification of Diseases (ICD-10) emphasizes the collection of more data on deaths, with the aim of classifying all deaths by underlying rather than direct causes. For example, the causes of death can be divided into three categories:

1. Immediate cause of death - Disease, injury or complication that causes death. E.g. bronchopneumonia.

2. Antecedent cause of death - Morbid conditions, if any, giving rise to the above cause. E.g fractured femur.

3. Contributory cause of death - Contributing to the death but not related to the disease condition causing it. e.g emphysema.3

However, such data is lacking in the context of ED in Nepal. Research on the causes of ED death will help improve the efficiency of ED and provide timely intervention to prevent death. Therefore, we attempted to conduct this study to determine the immediate, antecedent and contributory causes of mortality in ED at the Tribhuvan University Teaching Hospital as recorded in the death certificate.

\section{METHODS}

This was a retrospective study carried out in the adult emergency services of Tribhuvan University Teaching Hospital, Kathmandu. Data collected from death certificate for a period of six months between October 2017 to March 2018 were analyzed. The study was approved by Institutional Review Committee of Institute of Medicine. Information on age, gender, causes of death, and time of arrival to death were extracted from the records. All the patients who died after registration into emergency services were included in the study while those who had incomplete registration and brought dead were excluded.

The death certificate of the hospital categorizes cause of a death into three different categories:

1. Immediate cause of death - The disease, injury or complication which caused death. E.g. bronchopneumonia.

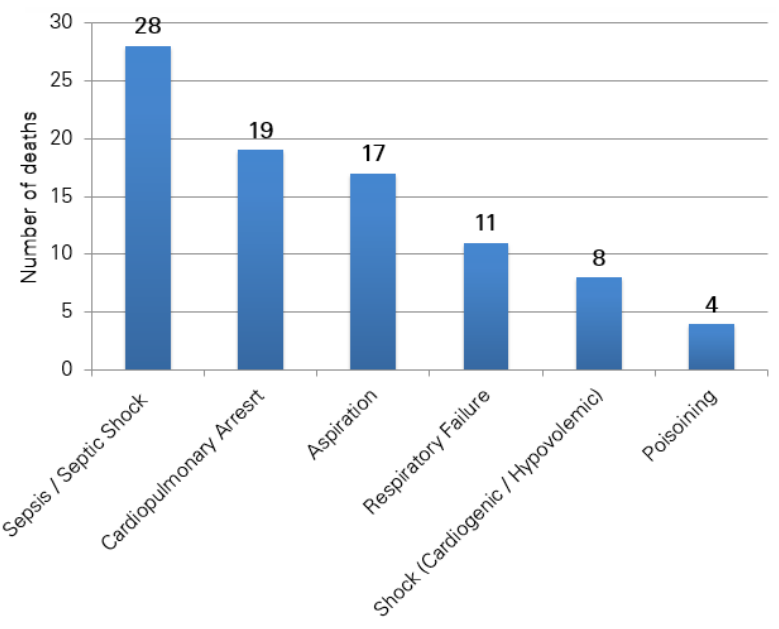

Figure 2. Immediate causes of mortality in Emergency

2. Antecedent cause of death - Morbid conditions, if any, giving rise to the above cause. E.g. fractured femur.

3. Contributory cause of death - Contributing to the death but not related to the disease condition causing it. E.g. emphysema.

\section{RESULTS}

An average of 150 patients per day attended the emergency services accounting a total of 26,982 patients taking emergency service during the study period. Among those patients, 847 patients with critical illness requiring intensive care were received in the red area. It accounts $3.1 \%$ of total patients seeking emergency services. A total of 128 patients died in the emergency accounting for $0.5 \%$ of total patients and $15.1 \%$ of critically ill patients. Male patients were slightly higher compared to female with male to female ratio 1.1:1.

Age of mortality cases ranged from 16 years to 97 years with mean age of 57.6 years. Age group 66-75 years had the highest i.e. about one-fourth (24.2\%) of total mortalities in the emergency. Age groupof 1625 years had least mortalities accounting $7.0 \%$ of the total deaths.

Sepsis/septic shock was the leading immediate cause of mortality (21.9\%) followed by cardio-pulmonary arrest, aspiration, respiratory failure, other causes of shock and poisoning.

Trauma was attributed as immediate cause of death in $10.9 \%$ of mortality cases.

In $21.1 \%$ death certificates, contributory causes were not mentioned and assumed to be absent. Chronic obstructive pulmonary disease was the most common contributory cause of death.

While 21 (16.4\%) patients were pronounced dead within 1 hour of arrival in the emergency room and a total of $63(49.2 \%)$ died within 6 hours of arrival. Twenty (15.6\%) patients died in the emergency after 


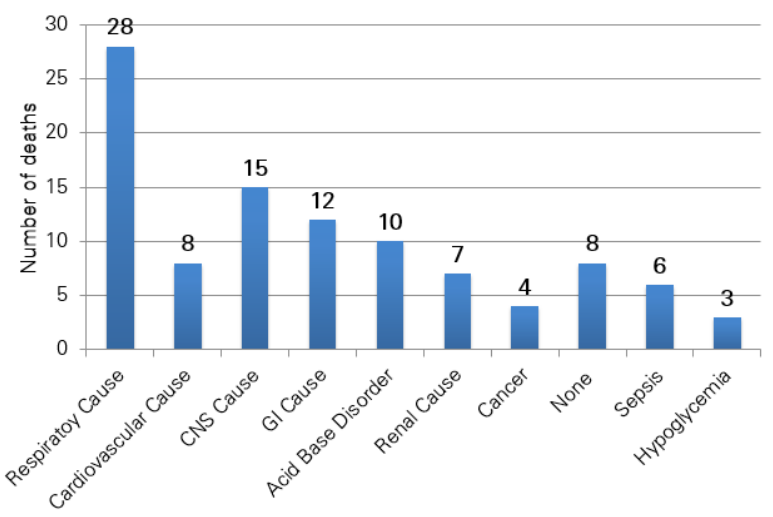

Figure 3. Antecedent causes of mortality

24 hours of arrival.

\section{DISCUSSION}

In this study, the male mortality rate was slightly higher than female with the M: F ratio of 1.1:1. In a similar study conducted in the same hospital 10 years ago, $\mathrm{M}$ : $\mathrm{F}$ ratio was 1:1.2. ${ }^{4}$ Male preponderance was even higher in other studies. ${ }^{2,5-7}$ The mean age of mortality cases in the study was 57.6 years, the age group of 66-75 years had highest mortality (24.2\%). The average age of death in our study was higher than the study conducted previously in Nepal and Ethiopia. ${ }^{4,8}$ On the other hand, it is less than the similar study done in the United States, Bulgaria and Iran. 2,6,7,9 During the study period, there were 128 deaths in the emergency department, accounting for $0.5 \%$ of the total number of patients. In a similar study in Nepal, the mortality rate was $0.3 \%$ to $0.4 \% .4,10$

In this study, sepsis/septic shock was the leading cause of immediate death (21.9\%). Interestingly, in previous studies, hemorrhagic shock was the leading cause of immediate death. ${ }^{4}$ Alimohammadi et al. found that cardiovascular disease $(39.2 \%)$ is the most common cause of immediate death in ED patients. ${ }^{2}$ The cardiac cause of mortality in this study was negligible because the institute has a separate heart center and emergency services in the same complex. In our study, cardiopulmonary arrest was recorded as the second most common immediate cause of death. The hospital's death certificate clearly states that deaths such as heart failure or respiratory failure should not be recorded as cause of death. However, the practice of reporting cardiopulmonary arrest as an immediate cause of death is still in practice. Errors in death certificates have been reported not only from Nepal ${ }^{11}$ but also from other parts of the world. ${ }^{6,12,13}$ Trauma (10.9\%) was the third most common immediate cause of death. A report from UK showed that trauma accounted for $14.2 \%$ of death in ED. Similarly, a report by Cummings et al. from USA reported that trauma was responsible for $23 \%$ of death in ED. ${ }^{5,6}$

Regarding antecedent causes of death, respiratory

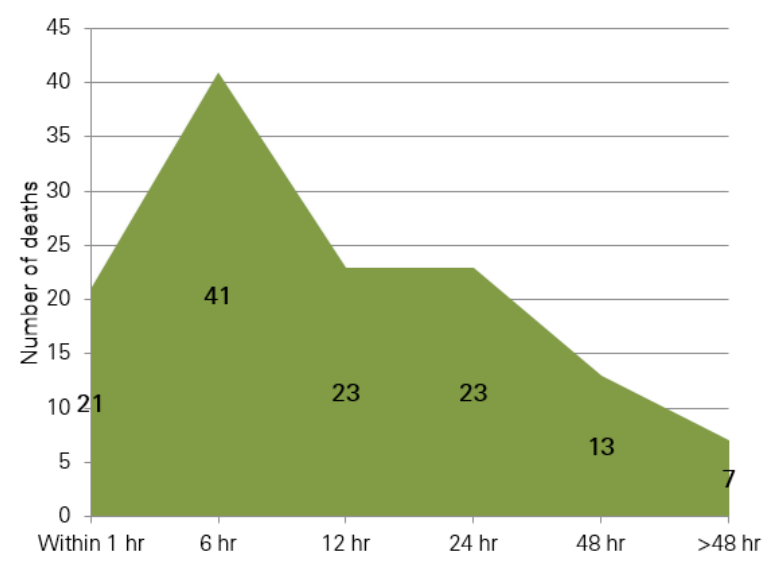

Figure 4. Time of mortality since arrival

disease was the most common cause (21.9\%) followed by central nervous system (CNS) disease, gastrointestinal disease, acid base disorder and cardiovascular disease. Chronic obstructive pulmonary disease (COPD) was the commonest contributory cause followed by chronic kidney disease, hypertension, diabetes mellitus and chronic liver disease. Alimohammadi et al. reported hypertension as most common contributory cause of death in ED. ${ }^{2}$ Gunnarsdottir OS et al. reported neoplasm as the leading contributory cause of death in ED. ${ }^{14}$ The above reported difference in causes of death are mainly due to geographical differences, differences in hospital settings, and differences in service availability.

The mortality rate in the first hour of an emergency visit was $16.4 \%$, and the mortality rate with in 6 hours arrival was $48.4 \%$. A similar study conducted at another tertiary hospital in Kathmandu demonstrated a mortality rate of $48.4 \%$ within 6 hours..$^{15}$ Most deaths $(84.4 \%)$ occurred within 24 hours of arrival, which is consistent with other studies. ${ }^{9,10}$ Since most deaths occur within 24 hours of arrival, timely placement of patients in relevant wards (including intensive care units) may help to minimize mortality. A study from Israel showed a direct relationship between emergency stay and mortality in the ED. ${ }^{16}$

Our research has some limitations. First, this is a retrospective study. Second, the emergency services at the Tribhuvan University Teaching Hospital do not manage cardiac emergencies. For a cardiac emergency, we have a different center. Therefore, the results must be interpreted with caution.

\section{CONCLUSION}

Sepsis and septic Shock were the most common immediate cause of death. Respiratory diseases were most common antecedent causes of death. Chronic obstructive pulmonary disease was the commonest contributory cause. Errors in death certificates need attention, including proper training of medical personnel, not only for effective research 
on mortality, but also for public health planning.

\section{ACKNOWLEDGEMENTS}

We would like to express my appreciation to the Department of General Practice and Emergency Medicine, Tribhuvan University Teaching Hospital for encourage and guidance during this research.

\section{CONFLICT OF INTEREST}

None declared.

\section{REFERENCES}

1. Olsen JC, Buenefe ML, Falco WD. Death in the emergency department. Ann Emerg Med. 1998 Jun;31(6):758-65.

2. Alimohammadi $\mathrm{H}$, Bidarizerehpoosh $\mathrm{F}$, Mirmohammadi $\mathrm{F}$, Shahrami A, Heidari K, Sabzghabaic A et al. Cause of Emergency Department Mortality; a Case-control Study. Emerg(Tehran).2014 Winter; 2(1): 30-35.

3. Randall B. Death certification: a primer. Part I-an introduction to the death certificate. S D Med. 2014, May;67(5):196-7,199.

4. R.K. Maharjan. Mortality Pattern in the Emergency Department in Tribhuvan University Teaching Hospital, Kathmandu, Nepal. Prehospital and DisasterMedicine. 2011May;26,(S1):160

5. Mushtaq F, Ritchie D. Do we know what people die of in the emergency department?.Emerg Med J. 2005 oct;22(10):718-21.

6. Peter Cummings. Cause of Death in Emergency Department. Am J Emerg Med. 1990 Sep;8(5):379-84.

7. Stefanovski PH, Vladimir Radkov R, Lyubomirllkov $\mathrm{T}$,
PenchoTonchev T, YoanaMladenova T, ViharManchev $\mathrm{K}$ etal. Analysis of mortality in the emergency department at a university hospital in Pleven. J Int Med Res. 2017 Oct; 45(5):1553-1561.

8. Hunchak C, Teklu S, MeshkatN,Meaney C, Puchalski Ritchie L. Patterns and predictors of early mortality among emergency department patients in Addis Ababa, Ethiopia. BMC Res Notes. 2015 Oct24;8:605.

9. Faridaalaee G, Nikzad F, Rahmani SH. Causes of Death in Emergency Department; a Brief Report. Iranian Journal of Emergency Medicine. 2015;2(1)

10. Shakya R P, Adhikari $S$, Munakarmi S. Mortality pattern in Emergency Department of a Tertiary care center in Western Nepal. Journal of Nobel Medical College. 2017Jul-Dec; 6(11):48-52.

11. Maharjan L, Shah A, Shrestha KB, Shrestha G. Errors in cause-of-death statement on death certificates in intensive care unit of Kathmandu, Nepal. BMC Health Serv Res. 2015 Nov 12;15:507.

12. Burger $E H$, van der Merwe $L$, Volmink J. Errors in the completion of the death notification form. S Afr Med J. 2007 Nov;979(11):1077-81.

13. QaddumiJA,Nazzal Z, Yacoup AR, Mansour M. Quality of death notification forms in North West Bank/Palestine: a descriptive study. BMC Res Notes. 2017 Apr 11;10(1):154.

14. Gunnarsdottir OS, Rafnsson V. Mortality of the users of a hospital emergency department. Emerg Med J. 2006 Apr;23(4):269-73.

15. Bharati $U$, Shrestha S. Mortality in Emergency Department of Nepal Medical College Teaching Hospital, a tertiary care centre in Kathmandu, Nepal. JIOM. 2017 April;39(1):90-94.

16. Raviv B, Israelit SH. Increased mortality of delayed patients in the emergency department of a tertiary medical center. Harefuah. 2015 Nov;154(11):697-700. 